\title{
BMJ Open Caregiver burden and associated factors among primary caregivers of patients with ALS in home care: a cross-sectional survey study
}

\author{
Shan Tang, ${ }^{1,2} \mathrm{Li} \mathrm{Li},{ }^{2}$ Hongxia Xue, ${ }^{2}$ Shuyan Cao, ${ }^{2}$ Chao Li, ${ }^{1}$ Kunjing Han, ${ }^{1}$ \\ Binquan Wang (iD ${ }^{1}$
}

To cite: Tang S, Li L, Xue H, et al. Caregiver burden and associated factors among primary caregivers of patients with ALS in home care: a cross-sectional survey study. BMJ Open 2021;11:e050185. doi:10.1136/ bmjopen-2021-050185

- Prepublication history for this paper is available online. To view these files, please visit the journal online (http://dx.doi. org/10.1136/bmjopen-2021 050185).

Received 12 February 2021 Accepted 24 August 2021

Check for updates

(c) Author(s) (or their employer(s)) 2021. Re-use permitted under CC BY-NC. No commercial re-use. See rights and permissions. Published by BMJ.

${ }^{1}$ College of Nursing, Shanxi Medical University, Taiyuan, Shanxi, China

${ }^{2}$ Department of Neurology, First Hospital of Shanxi Medical University, Taiyuan, Shanxi, China

Correspondence to Professor Binquan Wang; wbq_xy@126.com

\section{ABSTRACT}

Objectives This study aims to understand the caregiver burden experienced by the primary caregivers of patients with amyotrophic lateral sclerosis (ALS), and to explore the factors influencing caregiver burden.

Design A cross-sectional survey design was used. Setting This study was conducted with ALS inpatients and follow-up outpatients at the neurology department of a tertiary general hospital in Taiyuan, Shanxi, China and their caregivers.

Participants Patients with ALS and their caregivers ( $\mathrm{N}=120$ pairs) participated in a face-to-face interview. Primary and secondary outcome measures Primary outcome measures included the Zarit Burden Interview scores and personal/role burden scores. There were no secondary outcomes.

Results Multiple linear and logistic regression analyses were performed to examine the factors influencing burden in ALS patient's caregivers. Multiple linear regression showed that caregivers with higher Anxiety Index (Al) experienced greater personal $(\beta=0.089, p<0.001)$, role $(\beta=0.065, p<0.001)$ and overall $(\beta=0.200, p<0.001)$ burden. Logistic regression analysis showed that $\mathrm{Al}$ $(p=0.025$; OR 1.351, 95\% Cl 1.038 to 1.759$)$ and disease knowledge level $(p=0.033$; OR $0.305,95 \% \mathrm{Cl}$ 0.107 to 0.593 ) are the influencing factors of ALS load classification.

Conclusions Higher Al scores were associated with greater caregiver burden. Caregiver burden of caregivers who had no knowledge of the patient's disease was 0.305 times that of those who had good knowledge. The level of disease knowledge and Al score can serve as key predictors of caregiver burden in ALS.

\section{INTRODUCTION}

Amyotrophic lateral sclerosis (ALS) is an idiopathic and fatal neurodegenerative disease affecting the upper and lower motor neurons. It is a rapidly progressing disease, with no effective treatment so far; patients often die within 3-5 years of disease onset. ${ }^{12}$ Therefore, the treatment offered to patients with ALS is mainly palliative care, with a focus on symptom management, which aims
Strengths and limitations of this study

- Face-to-face interviews were conducted with patients with amyotrophic lateral sclerosis (ALS) and their caregivers.

- Several reliable and valid standardised instruments were used for data collection.

- The limited sample size of the survey reduced the ability to conduct a more in-depth investigation of the factors influencing ALS caregiver burden.

- As the measurement scales were based on selfratings, the subjective emotional interference of the participants may have led to recall bias.

to maximise the quality of life of patients and caregivers and minimise the burden of disease. $^{3}$

Most patients with ALS receive home care, and their caregivers are often their partners and children. Long-term care provision may affect caregivers' work performance and life, and they are likely to experience symptoms such as fatigue and sleep-related problems. ${ }^{4}$ An average of two caregivers are required by each patient with ALS, ${ }^{5}$ and the average time dedicated to care per day is 9.5 hours, most of which is spent on housework, and feeding and grooming the patient. As the disease progresses, worsening symptoms may induce increased caregiver stress, worry and burden, preventing them from enjoying their own leisure activities and taking care of their own needs. ${ }^{6}$ Furthermore, when the disease progresses to a more critical stage, patients' respiratory function is often impaired and mechanical ventilation is required, which has been shown to result in even heavier caregiver burden an average of 14.4 hours of care and 2.4 wakeups per day and night, respectively. ${ }^{7}$ Such burdens lead $30 \%$ of caregivers to believe that their own quality of life is inferior to that of the 
patient's. Additionally, a Japanese study ${ }^{8}$ suggests that caregiver burden for ALS is higher than that for other chronic diseases, such as Parkinson's and multiple system atrophy.

In addition to time and energy, caregivers have to witness the fatal disease and bear the increasingly heavy responsibilities. Moreover, they are riddled with endless worry and horror about the future resulting in psychological struggle. ${ }^{910}$ Caregivers have also been found to suffer from anxiety and depression, which are correlated with each other. ${ }^{11}$ The patient's worsening condition may lead to the corresponding deterioration of caregiver's mental health and the quality of life, and they are more likely to feel anxious and depressed due to the increasing care burden. ${ }^{12}$ Furthermore, some even need to take antidepressant drugs. ${ }^{13}$

Therefore, it is important to study the caregiver burden of primary caregivers of patients with ALS and its influencing factors. Nevertheless, studies in China and abroad on this topic are limited, with most having used univariate analyses, and lacking in-depth analysis. This study investigated the caregiver burden and its influencing factors among primary caregivers of patients with ALS receiving home care in China, to generate evidence to effectively reduce the burden of caregivers and formulate scientific intervention measures.

\section{MATERIALS AND METHODS \\ Participants}

Convenience sampling was used to recruit 124 pairs of patients with ALS and their primary caregivers. The patients were inpatients and follow-up outpatients at the neurology department of a tertiary general hospital in Taiyuan, Shanxi between January 2019 and May 2020.

\section{Inclusion and exclusion criteria for patients with ALS}

Inclusion criteria: patients with ALS must (1) meet the El Escorial World Federation of Neurology criteria for ALS revised in $1998^{14}$ and (2) give informed consent to participate in the study. Exclusion criteria: patients with (1) serious mental illness and cognitive impairment or (2) comorbid severe organ failure, malignant tumours and other serious diseases.

\section{Inclusion and exclusion criteria for caregivers for patients with ALS}

Inclusion criteria: Caregivers must (1) be relatives of the patient (including spouse, parent, child, son or daughterin-law, sibling, etc); (2) be responsible for primary caregiving duties during and after the patient's hospitalisation; (3) have basic communication and reading skills and (4) provide informed consent to participate in the study. Exclusion criterion: (1) Individuals with severe mental illness and cognitive impairment who were unable to complete the questionnaire.

\section{Instruments}

\section{General information questionnaire}

A general information questionnaire was developed, including the patient's general information (eg, gender, age, education level, marital status, healthcare payment methods, disease duration) and the caregiver's general information (eg, gender, age, education level, marital status, relationship with patient, employment status, health status, economic status, place of residence, average daily time of care, years of care, level of disease knowledge).

\section{ALS Functional Rating Scale-Revised}

The ALS Functional Rating Scale-Revised (ALSFRS-R) ${ }^{15}$ was used to assess the functional level of patients with ALS through 12 items: speech clarity, salivation, swallowing, handwriting, handling utensils, dressing and hygiene, turning in bed and adjusting bedding, walking, climbing stairs, dyspnoea, orthopnoea and use of assisted mechanical ventilation. Each item is rated on a 5-point scale (0-4 points; total possible score $=48$ points). Lower scores indicate more severe functional impairment. Disease severity is categorised into mild (37-48 points), moderate (25-36 points) and severe (0-24 points). In Dan Geng's study, Cronbach's $\alpha$ coefficient of the ALSFRS-R for the Chinese ALS population was $0.88 .{ }^{12}$

\section{Zarit Burden Interview}

The Zarit Burden Interview (ZBI) ${ }^{16}$ was used to assess the level of burden experienced by caregivers of patients with ALS through five aspects of the caregiver: physical health, mental status, financial status, social life, and overall assessment. It comprises 22 items, divided into personal strain (12 items), role strain (6 items), independent items (3 items) and overall burden perceived by the caregiver ( 1 item), scored on a 5 -point scale ('Never' $=0$, 'Rarely' $=1$, 'Sometimes'=2, 'Quite Frequently'=3, 'Nearly Always' $=4$ ). Higher scores indicate heavier caregiver burden, which is classified as follows: little or no burden ( $0-20$ points), mild to moderate burden (21-40 points), moderate to severe burden (41-60 points) and severe burden (61-88 points). The Chinese version of the ZBI to evaluate the care burden of the chief caregivers of patients with Alzheimer's, disability and schizophrenia showed acceptable internal consistency, with Cronbach's $\alpha$ coefficient ranging from 0.87 to $0.903 .{ }^{17-19}$

\section{6-Item Short-Form Health Survey}

The 36-Item Short-Form Health Survey $\left(\right.$ SF-36) ${ }^{20}$ was used to assess the quality of life of patients with ALS and their caregivers. The scale comprises 36 items; one item is related to the indicators of health transition in the past year, while the other 35 can be divided into eight dimensions: physical functioning, role limitations due to physical problems, bodily pain, general health, vitality (VT), social functioning, role limitations due to emotional problems and mental health. The eight dimensions are classified into two major categories: physical component summary 
constituting physical problems, bodily pain and general health; and mental component summary constituting VT, social functioning, role limitations due to emotional problems and mental health. Each dimension is scored using its score calculation table; scores range from 0 (lowest quality of life) to 100 (highest quality of life). The scale is widely used in both domestic and international contexts. The Chinese version of the SF-36 questionnaire to measure the postsurgical quality of life of patients with arthritis and cervical spondylosis showed a Cronbach's $\alpha$ coefficient of $0.76-0.93 .^{21} 22$

\section{Self-rating Anxiety Scale}

The Self-rating Anxiety Scale (SAS) ${ }^{23}$ was used to measure the anxiety level of ALS patients' caregivers. The scale comprises 20 items scored using a 4-point scale ('Never or a little of the time' $=1$, 'Some of the time' $=2$, 'Good part of the time' $=3$, 'Most or all of the time' $=4$ ). The total score ranges from 0 to 80 ; higher scores indicate more severe anxiety. The total scores were converted into an Anxiety Index (AI) $(<50=$ no anxiety, $50-59=$ mild anxiety, $60-69=$ moderate anxiety and $\geq 70=$ severe anxiety). The SAS for assessing anxiety in preschool children's caregivers has demonstrated good internal consistency, with a Cronbach's $\alpha$ coefficient of $0.890 .{ }^{24}$

\section{Self-Rating Depression Scale}

The Self-rating Depression Scale (SDS) ${ }^{25}$ was used to measure the depression level of ALS patients' caregivers. It comprises 20 items scored using a 4-point scale based on the same method as the SAS. Higher scores indicate more severe depression $(<53=$ no depression, $53-62=$ mild depression, 63-72=moderate depression and $\geq 73=$ severe depression). The SDS for assessing depression in preschool children's caregivers has demonstrated good reliability, with a Cronbach's $\alpha$ coefficient of $0.919 .^{24}$

\section{Procedure}

Two investigators with standardised training conducted face-to-face surveys with patients with ALS and their primary caregivers. The survey included the general information questionnaire, ALSFRS-R, ZBI, SF-36, SAS and SDS. A set of valid questionnaires comprised fully filled out questionnaires by both the patient and their caregiver. Out of the 124 questionnaires distributed, 120 valid questionnaires were returned (response rate: $96.77 \%$ ). In the course of the investigation, we obtained written informed consent from all the persons under investigation.

\section{Statistical analyses}

Data processing and analysis were performed using SPSS V.22.0 (IBM) for Windows. The Kolmogorov-Smirnov test was performed to confirm the normality of measurement data. Normally distributed data were described using mean $\pm \mathrm{SD}$; between-group differences were tested using t-test or analysis of variance. Count data were described using frequency (composition ratio); between-group differences were tested using $\chi^{2}$ test. Multiple linear regression analysis was performed to examine the factors influencing the different subscale scores and the total score of the caregivers' self-perceived burden. Ordinal logistic regression was performed on the factors influencing the classification of self-perceived burden. The significance level was set at two tailed. A $p<0.05$ indicated statistical significance.

Family members of patients with ALS were recruited as participants. All participants signed written informed consent forms and volunteered to participate in the study. The study data were collected via a face-to-face questionnaire survey conducted between the researcher and the study participants.

\section{Patient and public involvement}

Patients or the public were not involved in the design, conducting, reporting, or dissemination plans of our research.

\section{RESULTS \\ General information of patients with ALS and primary caregivers}

A total of 120 patients and their caregivers were enrolled in this study. Among caregivers, the male to female ratio was 1:1.4; the mean age was $50.23 \pm 8.45$ years; most were married $(83.3 \%, 100 / 120) ; 80(66.7 \%)$ caregivers were the patients' spouses; more than $95 \%$ had average to good health; most caregivers $(91.7 \%, 110 / 120)$ did not employ care workers or nannies; most had a monthly income less than RMB3000/month; 62.5\% (75/120) lived in urban areas, the average care time was 4-8 hours per day; the distribution of the years of care provided was relatively dispersed; and most had at least some knowledge of the patient's disease. Among patients, the male to female ratio was $1: 1$, their average age was $52.21 \pm 5.58$ years, their education level was mostly primary to junior middle school, $87.5 \%$ (105/120) were married, $62.5 \%$ $(75 / 120)$ had medical insurance, and the average disease duration was 2.5 years (range: $1-3$ years).

\section{Analysis of factors influencing the caregiver burden score of caregivers of patients with ALS \\ Univariate analysis}

Univariate analyses were performed using the caregivers' total ZBI score and their personal/role burden scores as the dependent variables, and the general information, SF-36, SAS, SDS and ALSFRS-R scores of the patients with ALS and their caregivers as the independent variables (table 1, only meaningful results were included).

The results indicate that: (1) for personal burden, the burden on general health status disease knowledge level is greater. Additionally, the burden is heavier when: the anxiety classification is higher, the function level classification is severe, the $\mathrm{AI} /$ disease severity score is higher and the VT score is lower. Moreover, all of them reported significant statistical differences $(p<0.05)$; (2) for role burden, male caregiver reported a higher burden, and the burden on general health status and 


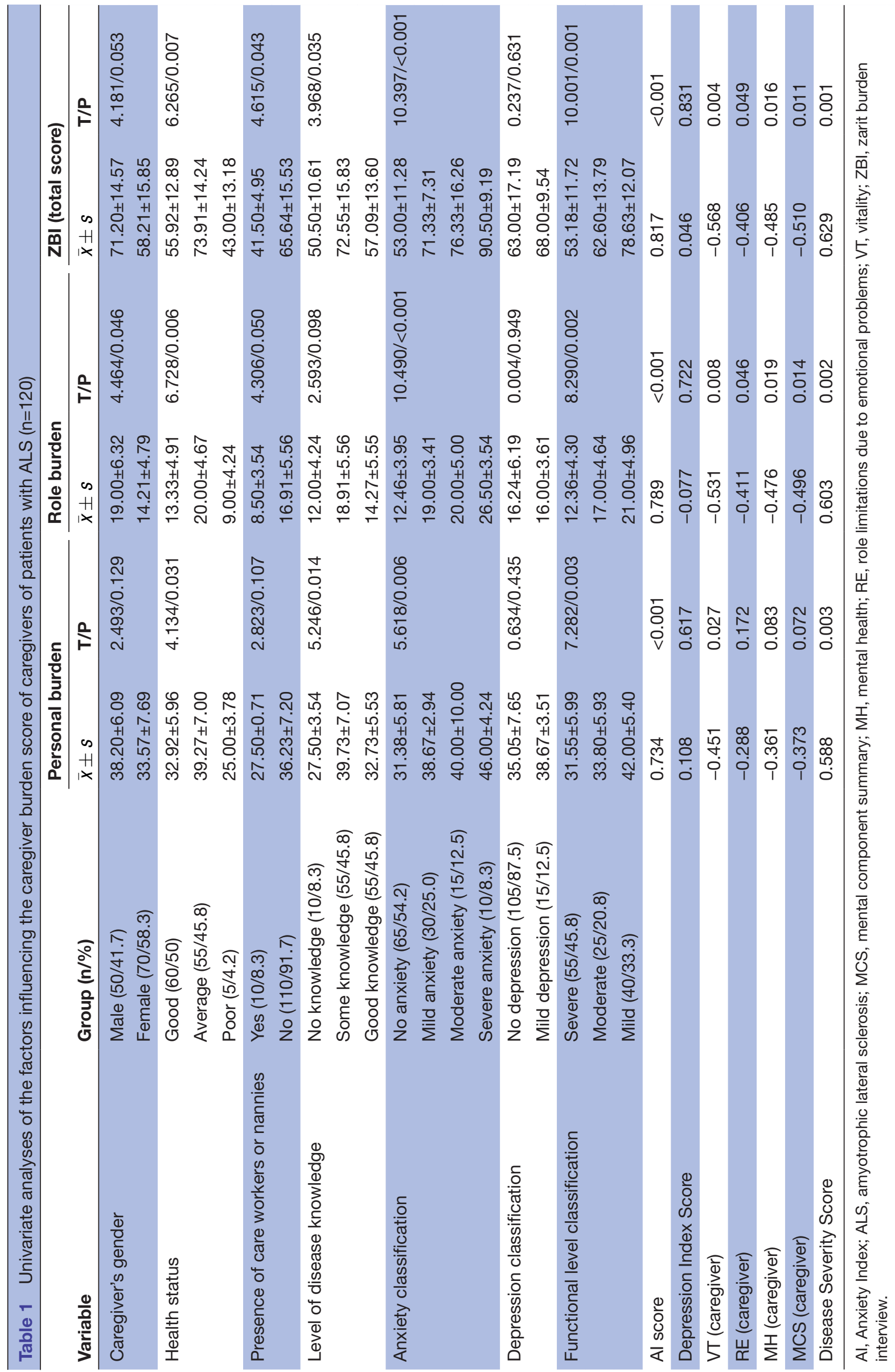


Table 2 Multiple linear regression analysis of factors influencing the caregiver burden score of caregivers of patients with ALS $(n=120)$

\begin{tabular}{|c|c|c|c|c|c|c|}
\hline ALS & $\begin{array}{l}\text { Independent } \\
\text { variables }\end{array}$ & $\begin{array}{l}\text { Regression } \\
\text { coefficients }\end{array}$ & SE & $\begin{array}{l}\text { Standardised regression } \\
\text { coefficient }\end{array}$ & T value & P value \\
\hline Personal strain & Constant & 12.987 & 4.556 & - & 2.851 & 0.009 \\
\hline \multirow[t]{2}{*}{ Role strain } & Constant & -3.205 & 3.315 & - & -0.967 & 0.344 \\
\hline & Al score & 0.389 & 0.065 & 0.789 & 6.014 & $<0.001$ \\
\hline
\end{tabular}

Confounding factors: health status, presence of care workers or nannies, level of disease knowledge, anxiety classification, functional level classification.

$\mathrm{Al}$, anxiety index; ALS, amyotrophic lateral sclerosis.

disease knowledge level is greater, and the presence of care workers or nannies is less of a burden. Furthermore, the burden is heavier when: the anxiety classification is higher, the function level classification is severe, the AI/ disease severity score is higher, and the VT/RE/MH/ MCS scores are lower. Moreover, all of them reported significant statistical differences $(p<0.05)$; (3) for the total burden score, the burden on general health status and disease knowledge level is greater and the presence of care workers or nannies is less of a burden, Additionally, the burden is heavier when: the anxiety classification is higher, the function-level classification is severe, the $\mathrm{AI} /$ disease severity score is higher, and the VT/RE/MH/ MCS scores are lower. Moreover, all of them reported significant statistical differences $(\mathrm{p}<0.05)$.

\section{Multiple linear regression analysis}

Multiple linear regression analysis was performed using the total ZBI score and subscale scores as the dependent variables, and statistically significant variables in the univariate analyses as the independent variables (table 2). After adjusting for confounding factors, the AI score was found to be an influencing factor of personal burden $\left(\mathrm{R}^{2}=0.518\right)$, role burden $\left(\mathrm{R}^{2}=0.605\right)$, and total burden score $\left(R^{2}=0.755\right)$.
Analysis of factors influencing the caregiver burden classification of caregivers of patients with ALS Univariate analyses

Univariate analyses were performed using the caregivers' ZBI burden classification as the dependent variable, and the general information, SF-36, SAS, SDS and ALSFRS-R scores of the patients with ALS and their caregivers as the independent variables (table 3, only meaningful results are included).

The proportion of severe burden for high knowledge level versus general knowledge level versus no relevant knowledge was $0.0 \%$ vs $90.9 \%$ vs $36.4 \%$, respectively, and the difference was statistically significant $\left(\chi^{2}=12.577\right.$, $\mathrm{p}<0.05)$. VI and disease severity scores reported that severe disease burden was higher than medium disease burden, which was higher than light disease burden, and the difference was statistically significant $(\mathrm{T}=8.352 / 5.305$, $\mathrm{p}<0.05)$. Similarly, the VT score of severe disease burden was higher than moderate disease burden, which was higher than mild disease burden, and the difference was statistically significant $(\mathrm{T}=4.198, \mathrm{p}<0.05)$.

\section{Ordinal logistic regression analysis}

Ordinal logistic regression analysis was performed using the ZBI burden classification as the dependent variable,

Table 3 Univariate analyses of caregiver burden classification of caregivers of patients with ALS ( $n=120)$

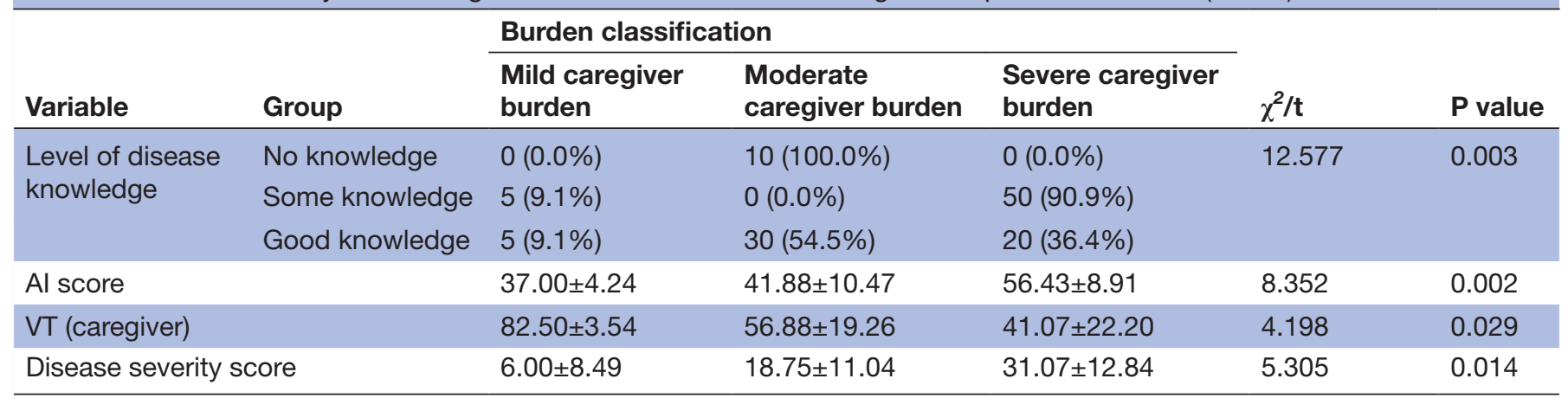

Al, anxiety index; ALS, amyotrophic lateral sclerosis; VT, vitality. 
Table 4 Ordinal logistic regression analysis of factors influencing the caregiver burden classification of caregivers of patients with ALS $(n=120)$

\begin{tabular}{lcccccc}
\hline & Estimate & SE & Wald $\chi^{2}$ & Df & P value & OR (95\% Cl) \\
\hline Al score & 0.301 & 0.135 & 5.005 & 1 & 0.025 & $1.351(1.038$ to 1.759$)$ \\
(Level of disease knowledge=no knowledge) & -6.692 & 3.1475 & 4.521 & 1 & 0.033 & $0.305(0.107$ to 0.593$)$ \\
(Level of disease knowledge=some knowledge) & 0.344 & 1.5671 & 0.048 & 1 & 0.826 & $1.410(0.065$ to 30.421$)$ \\
(Level of disease knowledge=good knowledge) & & & & & 1 \\
\hline
\end{tabular}

Al, Anxiety Index; ALS, amyotrophic lateral sclerosis.

and statistically significant variables in the univariate analyses and variables reported in the literature as the independent variables (table 4). After adjusting for confounding factors, AI score and level of disease knowledge were found to be influencing factors of ALS burden classification $(\mathrm{p}<0.05)$. More specifically, higher AI scores resulted in greater caregiver burden $(\mathrm{OR}=1.351,95 \% \mathrm{CI}$ : 1.038 to 1.759 ); the caregiver burden of those who had no knowledge of the patient's disease was $0.305(95 \%$ CI 0.107 to 0.593 ) times that of those who had good knowledge.

\section{DISCUSSION}

\section{Analysis of caregiver burden among caregivers of patients with ALS}

Caregiver burden refers to the feelings of loss, loneliness and other emotional changes perceived by caregivers due to the provision of home care, which comes at a physical, mental, emotional, social and economic cost to caregivers. It emphasises the negative outcomes resulting from the home care process. ${ }^{26}$ The findings show that the total caregiver burden score was $63.63 \pm 16.36$ points, which is classified as moderate to severe burden, and is higher than that reported in the USA, ${ }^{27}$ Italy $^{11}$ and India. ${ }^{28}$ There may be several reasons for this finding. First, in this study, $66.67 \%(80 / 120)$ of patients with ALS experienced moderate to severe impairment of physical function, only one-third of the patients could partially or completely perform self-care in daily life, and most patients required partial or full assistance from their caregivers. Therefore, the burden experienced by caregivers was moderate to severe, which was relatively high. Second, ALS undergoes rapid progression in the later stage, with the majority of patients dying within 3-5 years of onset, and generally presenting a short disease duration. In this study, the disease duration of patients with ALS was 1-3 years. During the rapid progression of this disease, patients with ALS gradually lose physical functioning and their care needs increase sharply, implying that caregivers need to spend more time and effort in caring for the patient. Finally, the inpatient treatment of patients with ALS is mainly carried out in tertiary general hospitals in China, the aim of which is to resolve the periodic presentation of problems such as dysphagia and respiratory dysfunction, thus causing relatively long hospital stays. This is inconsistent with the average length of hospital stay based on the performance appraisal indicators of tertiary public hospitals in China. Therefore, patients with ALS receive home care most of the time. Furthermore, standards of community rehabilitation in China are relatively poor, and its ability to provide treatment and care for critically ill patients is limited. In fact, most areas in China have yet to include ALS community treatment within the scope of medical insurance reimbursement. The medical insurance system in developed countries is more comprehensive, compared with which, patients with ALS and their caregivers in China may have greater economic burden. In addition, evidence-based, multidisciplinary palliative care models for patients with ALS have gradually been established in other countries, ${ }^{29-31}$ and the more comprehensive social support may have reduced caregiver burden to some extent.

\section{Analysis of factors influencing the caregiver burden of caregivers for patients with ALS \\ Level of disease knowledge}

Caregiver's knowledge of the disease was an influencing factor positively associated with caregiver burden. More specifically, the more knowledge the caregiver had of ALS, the heavier their caregiver burden. ALS is a fatal neurodegenerative disease. Patients tend to have a relatively late age of onset, generally around 60.7-64.3 years, and the time to diagnosis is $10.8-16.9$ months. ${ }^{32-35}$ Its clinical presentation includes progressive muscle atrophy, weakness and cramps, eventually leading to respiratory failure. More than $60 \%$ of patients die within 3 years of disease onset. ${ }^{1}$ In recent years, ALS has been known by the non-medical media in China as 'gradual freezing syndrome,' and has received growing attention. People are no longer limited to traditional hospitals and medical institutions for their health inquiry activities; caregivers of patients with ALS can easily access disease knowledge and health information through the Internet and smartphones, at every stage of the disease, from its onset and diagnosis to its progression. This allows them to have a clearer understanding about the rapid progression, physical paralysis, future care needs and eventual pain of losing their loved ones involved in ALS, increasing their caregiver burden. 


\section{Caregivers' state of psychological anxiety}

State of psychological anxiety was a factor that influenced the burden of caregivers. More specifically, caregivers with greater anxiety experienced heavier caregiver burden, while those experiencing greater caregiver burden were more prone to anxiety. This finding is consistent with the results of several studies in other countries. ${ }^{113637}$ ALS is a rapidly progressing disease with poor prognosis, in which patients often present with symptoms such as loss of speech and dysphagia in the later stages, eventually losing their self-care abilities and requiring caregivers to provide comprehensive care. In the later stages of ALS, some patients may display aggressive and obsessive-compulsive symptoms, and the caregiver burden resulting from cognitive and behavioural changes may be even greater than that caused by physical disability. ${ }^{38-40}$ Therefore, in the process of long-term home care, caregivers are faced with the difficulty of accepting the deterioration of their loved ones, while they may even need to give up their normal life and work, thus leading to changes in their family life and roles. This may have a serious impact on the mental health of caregivers, further exacerbating their caregiver burden.

\section{Severity of the disease}

International studies have shown that the severity of the disease in patients with ALS affects the degree of caregiver burden, ${ }^{14}{ }^{40-42}$ which is consistent with the univariate analysis results of this study. Most of the patients with ALS in this study were middle aged; $87.5 \%$ of them were married and bore a heavy financial burden and responsibilities. As the illness worsens, patients become more dependent on their caregivers; this in turn aggravates caregivers' negative emotions. Psychological disorders, such as anxiety and depression, in patients are strongly correlated with caregivers' anxiety and depression severity. ${ }^{43}$ The increasing negative emotions in patients increase the severity of family members' anxiety and caregivers' burden. As the disease continues to worsen, the psychological and care burden on caregivers should also be given more attention, to strengthen follow-up care services, and opportunities for communication should be created.

\section{Social support}

As a source of strength outside the family, social support can alleviate caregivers' pressure and help them regulate their emotions. The univariate analysis showed that in families with care workers or nannies, the burden of care is relatively low. However, in our study, the financial status of the family does not affect the burden of care, since most families in our sample reported a low monthly income level of less than RMB3000. Social support for families with patients with ALS is often reflected in how the medical bills are paid. Advances in medical care and treatment have brought about prolonged survival of patients; at the same time, higher medical expenses have also increased the pressure on families. Caregivers have to invest considerable time and energy into caregiving and bear not only caregiving-related pressure but also the accumulating financial burden. In China, the monthly cost of riluzole treatment for ALS is more than US $\$ 700$, unaffordable for Chinese people in view of their average income. Yet, this cost has not been covered by medical insurance. ${ }^{12}$ Therefore, society and communities should provide policy-related support for patients and their families as much as possible and reduce their financial burden through various measures, such as medical insurance, commercial insurance and community medical services, to improve patients' and their families' quality of life.

\section{Limitations}

This study has some limitations. First, the sample size is limited as all participants were recruited from a hospital in Shanxi province, China. Future studies should use larger sample sizes for more in-depth analysis. Cases from the more economically developed regions of central and eastern China and the less economically developed regions of western China could be investigated in further research. Second, as the measurement scales used were based on self-ratings, the subjective emotional interference of the participants may have led to recall bias. However, to minimise bias, the investigators were rigorously trained, the participants were given a detailed explanation of the survey purpose before filling out the questionnaires, and the questionnaires were completed in a quiet environment.

\section{CONCLUSION}

The caregivers of patients with ALS experienced moderate to severe levels of caregiver burden. Caregivers with more disease knowledge and greater psychological anxiety experienced greater caregiver burden. Thus, the level of disease knowledge and caregiver's state of psychological anxiety can serve as key predictors of caregiver burden in ALS.

These findings point to several measures that can help ease caregiver burden. Nurses should extend their clinical nursing practice from the hospital to home care. Additionally, group networks should be established for patients with ALS and their caregivers through multiple communication channels, to provide more care to caregivers of patients with ALS with severe symptoms in the later stages, guide caregivers to actively participate in the patient's symptom management, help analyse the patients' situation, and clarify the focal points of home care, thereby reducing the physical and mental burden experienced by caregivers. In terms of policy, there is an urgent need for a robust health insurance system and a comprehensive social support system. On the one hand, regional ALS centres can be established, staffed by teams comprising neurologists, physiotherapists, occupational therapists, speech pathologists, respiratory therapists, nutritionists, psychologists, assistive device experts, nurses and social workers. These teams can perform the comprehensive evaluation and timely observation of the patient's 
condition and help caregivers to jointly formulate and complete the patient's treatment plan, thus providing caregivers with a certain level of care support. On the other hand, ALS should be included within the scope of medical insurance reimbursement, which can partially alleviate the financial pressure on caregivers and protect their physical and mental health. Introducing interventional support at different levels can reduce the caregiver and economic burden of caregivers of patients with ALS, thereby promoting the physical and mental health of caregivers.

Acknowledgements We thank all caregivers of ALS patients for taking part in this research. We gratefully acknowledge the contribution of the doctors, nurses, and other staff members of the hospital.

Contributors Conceptualisation: ST and BW. Data curation: LL, HX, CL and KH. Formal analysis: LL and SC. Investigation: ST and HX. Methodology: ST and BW. Project administration: ST. Supervision: BW. Validation: ST. Writing-original draft: ST. Writing-review and editing: ST and BW.

Funding The authors have not declared a specific grant for this research from any funding agency in the public, commercial or not-for-profit sectors.

Competing interests None declared.

Patient and public involvement Patients and/or the public were not involved in the design, or conduct, or reporting, or dissemination plans of this research.

\section{Patient consent for publication Not applicable.}

Ethics approval This study was conducted from January 2019 to May 2020 and was approved by the Ethics Committee of the First Hospital of Shanxi Medical University (ethics approval no. (2020)|RB (K076)).

Provenance and peer review Not commissioned; externally peer reviewed.

Data availability statement Data are available upon reasonable request. The data that support the findings of this study are available from the corresponding author on reasonable request.

Open access This is an open access article distributed in accordance with the Creative Commons Attribution Non Commercial (CC BY-NC 4.0) license, which permits others to distribute, remix, adapt, build upon this work non-commercially, and license their derivative works on different terms, provided the original work is properly cited, appropriate credit is given, any changes made indicated, and the use is non-commercial. See: http://creativecommons.org/licenses/by-nc/4.0/.

\section{ORCID iD}

Binquan Wang http://orcid.org/0000-0002-3864-4093

\section{REFERENCES}

1 Kiernan MC, Vucic S, Cheah BC, et al. Amyotrophic lateral sclerosis. Lancet 2011;377:942-55.

2 O'Brien MR, Whitehead B, Jack BA, et al. From symptom onset to a diagnosis of amyotrophic lateral sclerosis/motor neuron disease (ALS/MND): experiences of people with ALS/MND and family carers a qualitative study. Amyotroph Lateral Scler 2011;12:97-104.

3 Connolly S, Galvin M, Hardiman O. End-Of-Life management in patients with amyotrophic lateral sclerosis. Lancet Neurol 2015:14:435-42.

4 Ray RA, Street AF. Non-finite loss and emotional labour: family caregivers' experiences of living with motor neurone disease. J Clin Nurs 2007;16:35-43.

5 Chiò A, Gauthier A, Vignola A, et al. Caregiver time use in ALS. Neurology 2006;67:902-4.

6 de Wit J, Bakker LA, van Groenestijn AC, et al. Caregiver burden in amyotrophic lateral sclerosis: a systematic review. Palliat Med 2018;32:231-45.

7 Kaub-Wittemer D, Steinbüchel Nvon, Wasner M, et al. Quality of life and psychosocial issues in ventilated patients with amyotrophic lateral sclerosis and their caregivers. J Pain Symptom Manage 2003;26:890-6.

8 Miyashita M, Narita Y, Sakamoto A, et al. Care burden and depression in caregivers caring for patients with intractable neurological diseases at home in Japan. $J$ Neurol Sci 2009;276:148-52.

9 Anderson $\mathrm{NH}$, Gluyas C, Mathers S, et al. "A monster that lives in our lives": experiences of caregivers of people with motor neuron disease and identifying avenues for support. BMJ Support Palliat Care 2019;9:e27.

10 Weisser FB, Bristowe K, Jackson D. Experiences of burden, needs, rewards and resilience in family caregivers of people living with motor neurone Disease/Amyotrophic lateral sclerosis: a secondary thematic analysis of qualitative interviews. Palliat Med 2015;29:737-45.

11 Pagnini F, Rossi G, Lunetta C, et al. Burden, depression, and anxiety in caregivers of people with amyotrophic lateral sclerosis. Psychol Health Med 2010;15:685-93.

12 Geng D, Ou R, Miao X, et al. Patients' self-perceived burden, caregivers' burden and quality of life for amyotrophic lateral sclerosis patients: a cross-sectional study. J Clin Nurs 2017;26:3188-99.

13 Rabkin JG, Albert SM, Rowland LP, et al. How common is depression among ALS caregivers? A longitudinal study. Amyotroph Lateral Scler 2009;10:448-55.

14 Brooks BR, Miller RG, Swash M, et al. El Escorial revisited: revised criteria for the diagnosis of amyotrophic lateral sclerosis. Amyotroph Lateral Scler Other Motor Neuron Disord 2000;1:293-9.

15 Cedarbaum JM, Stambler N, Malta E, et al. The ALSFRS-R: a revised ALS functional rating scale that incorporates assessments of respiratory function. BDNF ALS Study Group (phase III). J Neurol Sci 1999;169:13-21.

16 Zarit SH, Reever KE, Bach-Peterson J. Relatives of the impaired elderly: correlates of feelings of burden. Gerontologist 1980;20:649-55.

17 Zhong Y, Wang J, Nicholas S. Social support and depressive symptoms among family caregivers of older people with disabilities in four provinces of urban China: the mediating role of caregiver burden. BMC Geriatr 2020;20:3.

18 Li D, Hu N, Yu Y, et al. Trajectories of multidimensional caregiver burden in Chinese informal caregivers for dementia: evidence from exploratory and confirmatory factor analysis of the Zarit burden interview. J Alzheimers Dis 2017;59:1317-25.

19 Yu Y, Liu Z-wei, Li T-xin, et al. A comparison of psychometric properties of two common measures of caregiving burden: the family burden interview schedule (FBIS-24) and the Zarit caregiver burden interview (ZBI-22). Health Qual Life Outcomes 2020;18.

20 Ware JE, Sherbourne CD. The MOS 36-item short-form health survey (SF-36). I. conceptual framework and item selection. Med Care 1992;30:473-83.

21 Zhang Y, Zhou F, Sun Y. Assessment of health-related quality of life using the SF-36 in Chinese cervical spondylotic myelopathy patients after surgery and its consistency with neurological function assessment: a cohort study. Health Qual Life Outcomes 2015;13:39.

22 Rao Y, Xu X, Liu D, et al. Health-related quality of life in patients with arthritis: a cross-sectional survey among middle-aged adults in Chongqing, China. Int J Environ Res Public Health 2018;15:762.

23 Zung WW. A rating instrument for anxiety disorders. Psychosomatics 1971;12:371-9.

24 Qu G, Wang L, Tang X, et al. Association between caregivers' anxiety and depression symptoms and feeding difficulties of preschool children: a cross-sectional study in rural China. Arch Pediatr 2020;27:12-17.

25 Zung WW. A self-rating depression scale. Arch Gen Psychiatry 1965;12:63-70.

26 Zarit SH, Femia EE, Kim K, et al. The structure of risk factors and outcomes for family caregivers: implications for assessment and treatment. Aging Ment Health 2010;14:220-31.

27 Qutub K, Lacomis D, Albert SM, et al. Life factors affecting depression and burden in amyotrophic lateral sclerosis caregivers. Amyotroph Lateral Scler Frontotemporal Degener 2014;15:292-7.

28 Thomas PT, Warrier MG, Sadasivan A, et al. Caregiver burden and quality of life of patients with amyotrophic lateral sclerosis in India. Amyotroph Lateral Scler Frontotemporal Degener 2018;19:606-10.

29 Van den Berg JP, Kalmijn S, Lindeman E, et al. Multidisciplinary ALS care improves quality of life in patients with ALS. Neurology 2005;65:1264-7.

30 EFNS Task Force on Diagnosis and Management of Amyotrophic Lateral Sclerosis, Andersen PM, Abrahams S, et al. EFNS guidelines on the clinical management of amyotrophic lateral sclerosis (MALS)-revised report of an EFNS task force. Eur J Neurol 2012;19:360-75.

31 MacDermott CN, Maden S, McLoughlin HJ, et al. Review of 'palliative care in amyotrophic lateral sclerosis from diagnosis to bereavement' by David Oliver, Gian Domenico Borasio, Wendy Johnson. BMJ Support Palliat Care 2017;7:5-6.

32 Mehta P, Antao V, Kaye W, et al. Prevalence of amyotrophic lateral sclerosis - United States, 2010-2011. MMWR Supp/ 2014;63:1-13. 
33 Chiò A, Mora G, Calvo A, et al. Epidemiology of ALS in Italy: a 10-year prospective population-based study. Neurology 2009;72:725-31.

34 Traxinger K, Kelly C, Johnson BA, et al. Prognosis and epidemiology of amyotrophic lateral sclerosis: analysis of a clinic population, 19972011. Neurol Clin Pract 2013;3:313-20.

35 Traynor BJ, Codd MB, Corr B, et al. Incidence and prevalence of ALS in Ireland, 1995-1997: a population-based study. Neurology 1999;52:504.

36 Krivickas LS, Shockley L, Mitsumoto H. Home care of patients with amyotrophic lateral sclerosis (ALS). J Neurol Sci 1997;152 Suppl 1:s82-9.

37 Gauthier A, Vignola A, Calvo A, et al. A longitudinal study on quality of life and depression in ALS patient-caregiver couples. Neurology 2007;68:923-6.

38 Lillo P, Mioshi E, Hodges JR. Caregiver burden in amyotrophic lateral sclerosis is more dependent on patients' behavioral changes than physical disability: a comparative study. BMC Neurol 2012;12:156.

39 Marconi A, Meloni G, Fossati F, et al. Aggressiveness, sexuality, and obsessiveness in late stages of ALS patients and their effects on caregivers. Amyotroph Lateral Scler 2012;13:452-8.

40 Burke T, Elamin M, Galvin M, et al. Caregiver burden in amyotrophic lateral sclerosis: a cross-sectional investigation of predictors. $J$ Neurol 2015;262:1526-32.

41 Tramonti F, Bongioanni P, Leotta R, et al. Age, gender, kinship and caregiver burden in amyotrophic lateral sclerosis. Psychol Health Med 2015;20:41-6.

42 Tremolizzo L, Pellegrini A, Susani E, et al. Behavioural but not cognitive impairment is a determinant of caregiver burden in amyotrophic lateral sclerosis. Eur Neurol 2016;75:191-4.

43 Chen D, Guo X, Zheng Z, et al. Depression and anxiety in amyotrophic lateral sclerosis: correlations between the distress of patients and caregivers. Muscle Nerve 2015;51:353-7. 